\title{
La caída de los muros del conocimiento en la sociedad digital y las pedagogías emergentes
}

\section{The fall of the walls of knowledge in the digital society and the emerging pedagogies}

\author{
Begoña Gros \\ Facultad de Educación \\ Universidad de Barcelona \\ bgros@ub.edu \\ Fecha de recepción: 26-1-2015 \\ Fecha de revisión: 2-3-2015
}

\section{Palabras clave:}

\author{
Sociedad del Conocimiento; ubicuidad; entor- \\ nos personales de aprendizaje; pedagogías \\ emergentes; aprendizaje sin costuras.
}

\section{Resumen}

Este artículo sostiene que una de las consecuencias más importantes de la sociedad del conocimiento es la transformación de los espacios y lugares para el aprendizaje. Consideramos el aprendizaje "sin costuras", la ubicuidad y los entornos personales de aprendizaje como pilares fundamentales de los nuevos espacios formativos. Las pedagogías que emergen deben posibilitar la eliminación de los muros del conocimiento dotando a las personas de la capacidad suficiente para enfrentarse a un aprendizaje a lo largo y ancho de la vida. Para ello, sostenemos que es necesario el desarrollo de una pedagogía mucho más transparente y basada en el diseño del aprendizaje. En este artículo abordamos el significado actual del conocimiento y los cambios que suponen el lugar y los tiempos para el aprendizaje a través de la caracterización del aprendizaje. Finalmente, establecemos las características y dimensiones de las pedagogías que emergen para posibilitar la eliminación de los muros del conocimiento.
Fecha de aceptación: 20-3-2015

Fecha de publicación: 27-3-2015

Keywords:

\author{
Knowledge society; ubiquity; personal \\ learning networks; emergent pedagogies; \\ seamless learning.
}

\begin{abstract}
The contribution analyse the consequences of knowledge society in the transformation of the space and place for learning. We consider that there are three main pillars in the new learning spaces that we have to take into account: the seamless learning, the ubiquity and the personal learning networks. The emergent pedagogies have to facilitate the fall of the walls of the knowledge. It is necessary to provide new skills for lifelong learning and wide learning. We consider that it is necessary the development of new pedagogies much more transparent and based on learning design. This article deals with the actual meaning of knowledge and the changes involved the place and time for learning through the characterization of learning. Finally, we establish the characteristics and dimensions of emerging pedagogies to enable the removal of the walls of knowledge.
\end{abstract}




\section{Introducción}

Suárez (2012) señala que el espacio tradicional para aprender y enseñar pasa "de ser un espacio-lugar a ser un espacio-nodo de la red de interacción global. La noción educativa de lugar de aprendizaje no desaparece, sino que el concepto se mueve en otra cartografía reticular formando parte de ella como nodo, una posición angularmente diferente a la concepción de escuela como centro, lugar de saber". Los espacios de aprendizaje están formados por una red en la que se combinan los espacios físicos y los espacios virtuales fortaleciendo la idea del aprendizaje en cualquier momento $\mathrm{y}$ en cualquier lugar. Los dispositivos digitales facilitan la ubicuidad, nos acompañan, forman parte de nosotros y constituyen artefactos cognitivos y emocionales de gran relevancia para el aprendizaje. Desde el punto de vista de la persona, el reto es dar significado a las conexiones entre las experiencias, datos e informaciones obtenidas en los diferentes espacios y recursos. Desde el punto de vista del profesional de la educación, el reto es diseñar situaciones que permitan dar sentido y coherencia a los espacios facilitando las conexiones y, por consiguiente, el aprendizaje. Los muros han caído pero eso no significa que todo el mundo los atraviese sin dificultades, para movernos sin problemas y con libertad necesitamos estar preparados para enfrentarnos a una sociedad del rendimiento (Han, 2012) en el que el peso de la educación y el aprendizaje está en uno mismo.

En este artículo abordamos el significado actual del conocimiento y los cambios que supone el lugar y los tiempos para el aprendizaje a través de la caracterización del aprendizaje "sin costuras", la ubicuidad y los entornos personales de aprendizaje como pilares fundamentales de los nuevos espacios. Finalmente, establecemos las características y dimensiones de las pedagogías que emergen para posibilitar la eliminación de los muros del conocimiento.

\section{El sentido del conocimiento en la sociedad del conocimiento}

Es una obviedad afirmar que el conocimiento es una de las características básicas de la sociedad actual. Sin embargo, dicha obviedad es substancial ya que el hecho de que la sociedad esté vinculada a la producción del conocimiento altera las formas tradicionales de entender el propio concepto, su elaboración, expansión y, por supuesto, las organizaciones dedicadas a su creación y difusión. En el siglo $\mathrm{XX}$, la vida del conocimiento era medida en décadas. Hoy, se devalúa con rapidez, la vida media del conocimiento está disminuyendo y la expectativa de relevancia y validez se ha reducido en algunas disciplinas desde un ciclo de años y décadas a otro de meses y años (Siemens, 2006). Sin embargo, la sociedad del conocimiento no tiene que ver con el hecho de que se está produciendo cada vez más. No es un tema de cantidades sino del significado que le otorgamos. Hemos pasado de pensar en el conocimiento como algo objetivo, estable, producido por expertos y que se puede transmitir a algo subjetivo, dinámico y producido de forma colaborativa. El conocimiento no es una verdad objetiva sino variable y verificable. Por ello, está marcado por la disposición de poner en cuestión las percepciones, las expectativas tradicionales y socialmente aceptadas. Las reglas y evidencias de nuestra sociedad están cada vez más sometidas a procesos de reflexión. Como muy bien afirma Innerarity, la consideración de que una sociedad se basa en el conocimiento no depende de lo que se manifiesta en una certificación académica. "El saber no es algo que las personas tienen en propiedad. La gestión de los procesos de aprendizaje es más importante que la administración de los saberes" (Innerarity, 2011: 59).

El constructivismo social enfatizó la idea 
de que el conocimiento no solo se construye de forma individual en la mente del sujeto sino que hay una construcción dinámica y cambiante de origen social y cultural. La persona aprende a través de la internalización del conocimiento socialmente construido. Hacía falta conseguir un equilibrio entre lo subjetivo y lo objetivo de la noción conocimiento, para lo cual se puede recurrir a las propuestas conceptuales del pragmatismo norteamericano de Dewey (Dewey, 1975) y a la teoría de sistemas desarrollada por el sociólogo Luhmann (1998). Este último define el conocimiento como un esquema cognitivo que se considera verdadero, pero que, al mismo tiempo, es variable. El conocimiento no representa el mundo de forma objetiva pero hay un criterio para su adecuación (su verdad), que reside en su convalidación en la práctica aunque estos efectos prácticos no están dados de forma objetiva, sino que a su vez se constituyen a través de las interrelaciones entre las personas perceptoras y actuantes por un lado, y la realidad por otro lado. De esta forma, se construye socialmente una certeza de la realidad que es condición imprescindible para cualquier formar de pensar y de actuar. (Luhmann 1998: 166). Bereiter (2002) recupera las ideas de Dewey e intenta analizar cómo se produce el conocimiento a través de los sistemas sociales participativos derivados del uso de Internet. Este autor utiliza el concepto de producción de conocimiento para referirse a la idea de un trabajo colectivo para el avance y la elaboración de los artefactos conceptuales tales como las ideas, las teorías los modelos y las entidades. La construcción del conocimiento va más allá de la metáfora de la participación, las ideas, teorías e hipótesis son tratadas como artefactos culturales y objetos de investigación que pueden ser discutidos, mejorados y puestos en nuevos usos a medida que los participantes se comprometen en una progresiva investigación. Siemens (2010) plantea la conectividad como el aspecto clave en la producción del conocimiento en la sociedad actual.
"La conectividad permite que los individuos creen
y distribuyan sus propios materiales e identidad.
Ya no contemplamos un todo sino muchas piezas que componen el todo, y como individuos creamos una versión del todo que se adapta a nuestras necesidades e intereses. Cuando el conocimiento deja de existir en espacios físicos podemos duplicar (o conectar) entidades en espacios múltiples. El conocimiento, cuando es digital (no en entidades físicas como libros o revistas de papel) puede ser combinado (o remezclado) fácilmente con nuevo conocimiento. Combinar ideas de dos libros diferentes requiere de un esfuerzo para combinar las entidades (comprar los libros o ir a la biblioteca). Con el conocimiento digital, podemos enlazar y combinar ideas con facilidad" (Siemmens, 2010: 72).

Las actividades desarrolladas desde esta nueva dimensión de la red se configuran como culturas de aprendizaje permeables a la incorporación constante de nuevas ideas, tecnologías y tendencias de uso, que los propios usuarios, individual o colectivamente, se encargan de legitimar y de popularizar en el amplio espectro de la red (Jokisalo y Riu, 2009). Todo ello nos lleva a cuestionarnos si realmente podemos referirnos a los procesos formales e informales de aprendizaje como algo separado y distinto.

\section{Los espacios y tiempos del conocimiento}

La conectividad en la sociedad actual no solo ha alterado el sentido y la producción del conocimiento, sino también los espacios y los tiempos del aprendizaje, rompiendo la organización social del siglo XX. En esta línea, consideramos que hay tres conceptos importantes que han irrumpido en el ámbito del aprendizaje y que conviene tener en consideración, ya que constituyen los pilares básicos de la formación en la sociedad actual: el aprendizaje sin fisuras, la ubicuidad y los entornos personales de aprendizaje.

En el año 2012, aparece el informe Innovating Report de la Open University señalando como tendencia clara de innovación la denominada "seamlees learning" que literalmente es 
traducido como "aprendizaje sin costuras o sin fisuras". Este tipo de aprendizaje no es otro que el que se produce a través de diferentes contextos y que por tanto abarca no solo el aprendizaje a lo largo de la vida sino también a lo ancho de la vida. Los distintos espacios por los que nos movemos en un determinado momento. Sharples et al., (2012: 24) lo define como "el aprendizaje que se produce a través de diferentes contextos y forma parte de un viaje de aprendizaje más amplio que abarca las transiciones de la vida de una persona, desde la escuela a la universidad o el lugar de trabajo (...). Es cuando una persona experimenta una continuidad de experiencias de aprendizaje a través de diferentes contextos y tecnologías".

Las tecnologías móviles permiten a los estudiantes de todas las edades operar a través de diferentes contextos. Los alumnos pueden iniciar un tema de trabajo en clase, a continuación, realizar una recogida de datos en casa o al aire libre, elaborar un nuevo conocimiento con la ayuda del software, y realizar un intercambio de conocimientos en el aula o en un entorno virtual.

La noción del aprendizaje sin fisuras también está relacionada con el aprendizaje que surge de las experiencias obtenidas en los espacios virtuales. Nuestras actividades en línea están cada vez más adaptadas a nuestros intereses: las páginas de búsqueda ordenan las respuestas basadas en consultas anteriores; los sitios web recomiendan contenido relacionado con nuestra visualización previa, estableciendo vínculos entre nuestras acciones.

El hecho de que el conocimiento pueda ser un nodo de una red y que cualquier red pueda proporcionar conocimiento significa que todo y todos podemos ser recursos para el aprendizaje. Burbules (2014) señala que se puede aprender de todo y de todos. El aprendizaje se hace ubicuo, lo que afecta a los espacios de aprendizaje. La ubicuidad implica una especial capacidad para la flexibilidad y la adaptación a contextos diversos y en constante movimiento. Mientras que en un aula tradicional, el profesor es la principal fuente de información y los estudiantes están obligados a permanecer en el mismo lugar y participar simultáneamente en la misma actividad, en una situación de aprendizaje ubicuo las actividades pueden resolverse en un espacio-tiempo diferente para cada estudiante. Además, los materiales de enseñanza se encuentran disponibles en todo momento y son accesibles desde cualquier dispositivo. Burbules señala que para que el aprendizaje sea efectivamente "ubicuo" se requiere de una experiencia más distribuida en el tiempo y el espacio. Se entiende así que un ambiente de aprendizaje ubicuo es una situación o contexto educativo generalizado u omnipresente, en el que incluso el estudiante puede estar aprendiendo sin ser completamente consciente.

El aprendizaje ubicuo también se relacionada con las tecnologías móviles, ya que hace referencia al hecho de que cualquier persona gracias a las tecnologías digitales está conectada en cualquier momento y en cualquier lugar produciendo y difundiendo información.

La ubicuidad se apoya y se facilita a través de los dispositivos móviles que permiten generar entornos de aprendizaje a los que se puede acceder en diferentes contextos y situaciones. Cuatro características definen el aprendizaje ubicuo (Cope y Kalantzis, 2010):

- Permanencia: El usuario conserva la información y el trabajo realizado al pasar de un medio a otro. La traza es única, independiente del medio que se utilice.

- Accesibilidad: Los usuarios tienen acceso a los recursos formativos desde cualquier lugar y momento propiciando un aprendizaje auto-dirigido.

- Inmediatez: El usuario puede obtener y buscar toda la información de inmediato, cuando él lo decida.

- Interactividad: En todo momento, el usuario puede interactuar con expertos, profesores, o compañeros de forma síncrona o asíncrona.

La ubicuidad y el aprendizaje sin fisuras plantean a la persona la necesidad de crear espacios personales para poder tomar implícitamente el control de su propio aprendizaje. Los entornos personales de aprendizaje (PLEs) tratan de dar respuesta a las necesidades e intereses de aprendizaje permanente desde la perspectiva de los aprendices.

Bartolomé y Steffens (2011) proponen una serie 
de criterios que los entornos de aprendizaje enriquecidos por tecnología deben cumplir con el fin de apoyar los procesos de aprendizaje autorregulados: a) alentar a los estudiantes a planificar su propia actividad de aprendizaje, b) proporcionar retroalimentación sobre el desempeño de las tareas con el fin de facilitar su seguimiento y la correcta autodirección del proceso de aprendizaje y c) proporcionar a los estudiantes los criterios para evaluar los resultados de su aprendizaje. En su análisis, estos autores consideran al PLE como el único entorno capaz de cubrir los tres requisitos mencionados.

Como hemos visto previamente, Siemens plantea la conectividad como un aspecto clave en la producción del conocimiento en la sociedad actual (Siemens, 2010). El conocimiento personal se compone de una red, la cual alimenta a organizaciones e instituciones, que a su vez retroalimentan a la red, proveyendo de un nuevo aprendizaje a los individuos. Este ciclo de desarrollo del conocimiento (de las personas a la red, de la red a la institución) permite a los aprendices estar actualizados en su área y potenciar el aprendizaje a lo largo de la vida. El proceso de renovación y actualización del propio perfil de competencias de manera autorregulada y autodirigida puede requerir tomar parte en procesos de aprendizaje formal pero también fuera de estos. En este sentido, un PLE puede ser visto como una plataforma basada en las redes sociales, centrada en el aprendiz y diseñada para permitir compartir, colaborar y producir recursos y contenidos a través de procesos de participación distribuida. Si entendemos el aprendizaje como situado, debemos admitir que el aprendizaje apoyado por los PLEs supone un cierto nivel de descontextualización de la información, ya que esta se extrae de su contexto original y se recontextualiza en el PLE. Por ello, se requieren estrategias para reconocer qué información es relevante para ser integrada en el conocimiento actual y construir relaciones entre objetos, metas y dominios, extendiendo el contexto de aprendizaje más allá del lugar original. Desde esta perspectiva, un PLE puede desempeñar el papel de objeto frontera, que permite al aprendiz pasar de un dominio a otro, realizando conexiones entre los objetos de información sobre la base de las relaciones sociales que entabla (Atwell, 2010). Así, un PLE es un entorno creado en el mismo proceso de aprendizaje, que incluye herramientas, pero también objetos de información y conocimiento, así como a los miembros de la comunidad o comunidades involucradas (Wild, Mödritscher y Sigurdarson, 2008).

\section{Características de las pedagogías emergentes}

Hemos sostenido que los muros van cayendo y como señala Han (2012: 25), la sociedad disciplinaria de Foucault, que constaba de hospitales, psiquiátricos, cárceles, cuarteles y fábricas, ya no se corresponde con la sociedad actual. En su lugar se ha establecido una "sociedad del rendimiento". El sujeto del rendimiento no sufre una explotación externa: es dueño de sí mismo y se auto-explota. El sujeto auto-explotado hace del multitasking una práctica que "modifica radicalmente la estructura y economía de la atención. Debido a esto la percepción queda fragmentada y dispersa" (Han, 2014: 34).

La consecuencia de la caída de los muros significa que la responsabilidad y la "carga" sobre la formación y el aprendizaje recaen en el aprendiz. En este sentido, conviene repensar el significado de la educación y, muy especialmente, hay que tener presente que para que realmente la persona pueda dirigir sus procesos formativos requiere desarrollar importantes capacidades que no se generan de forma espontánea. La formación sigue siendo necesaria pero no solo orientada a lo disciplinar sino, especialmente, a capacitar a las personas para pasar por los distintos espacios sin tener una experiencia fragmentada, dispersa y un exceso de carga emocional y cognitiva.

La pedagogía precisa elaborar planteamientos que orienten en el diseño de estos nuevos espacios a través de experiencias basadas en la interacción social, la participación activa 
y los entornos complejos. Cuatro aspectos básicos centran las estrategias de formación: la personalización, el aprendizaje activo, el aprendizaje colaborativo y el aprendizaje autónomo o autodirigido.

La transformación de las escuelas en lugares de aprendizaje requiere liderazgo de las personas que pueden aceptar nuevos desafíos y aprovechar las oportunidades. El acceso ubicuo a la tecnología debe ser un objetivo fundamental que permitirá a los estudiantes y profesores aprovechar al máximo las ventajas de la tecnología disponible.

Las actividades desarrolladas desde esta nueva dimensión de la red se configuran como nuevas culturas de aprendizaje informal, permeables a la incorporación constante de nuevas ideas, tecnologías y tendencias de uso, que los propios usuarios, individual o colectivamente, se encargan de legitimar y de popularizar en el amplio espectro de la red (Jokisalo y Riu, 2009). Por el contrario, la mayor parte de instituciones educativas formales de cualquier nivel educativo, continúan basándose en un enfoque jerárquico de la enseñanza. Las redes de conocimiento mediadas por las tecnologías sociales penetran inevitablemente en estos contextos, pero lo hacen de forma disruptiva puesto que representan una noción radicalmente distinta de la creación y la distribución de conocimiento, que cuestiona directamente a la tradicional. En definitiva, es necesario repensar los aprendizajes básicos propios de la educación formal y preparar un proceso que capacite a las personas como aprendices a lo largo de la vida.

Las pedagogías emergentes surgen en los contextos de la sociedad del conocimiento en red. Se basan en laintegración de las tecnologías digitales, la exploración y la modificación de las pedagogías existentes y desarrollan nuevas propuestas teóricas y prácticas. Como afirma Adell y Castañeda (2012), las pedagogías emergentes no son necesariamente nuevas pedagogías, necesitan explorar y volver a examinar las pedagogías existentes, examinando su contribución en el contexto de la sociedad del conocimiento en red. Es necesario integrar los principios pedagógicos que proporcionan una mejor adaptación a las necesidades actuales de los alumnos en los sistemas educativos y la evaluación de su eficacia. La mayoría de los elementos de instrucción de las nuevas pedagogías no son "nuevas" estrategias de enseñanza; aunque diríamos que las asociaciones de aprendizaje activas con estudiantes son nuevos. Muchas de las estrategias de enseñanza que han sido defendidas hace más de un siglo por autores como Dewey, Piaget, Montessori y Vygotsky están comenzando a emerger. Seguramente, las condiciones para que estas ideas se afianzaran y prosperaran no existían. Hoy en día, hay indicios de que esto está cambiando. Estamos viendo una forma de contagio positivo ya que estas estrategias de enseñanza comienzan a afianzarse en las escuelas regulares.

La relación entre las pedagogías y las tecnologías es substancial. En este sentido, Veletsianos define las tecnologías emergentes como
"herramientas, conceptos, innovaciones y avances utilizados en diversos contextos educativos al servicio de diversos propósitos relacionados con la educación. Además, propongo que las tecnologías emergentes ("nuevas" y "viejas") son organismos en evolución que experimentan ciclos de sobre expectación y, al tiempo que son potencialmente disruptivas, todavía no han sido completamente comprendidas ni tampoco suficientemente investigadas." (Veletsianos 2010, 33).

Esta definición es compatible con la relación mutua entre las tecnologías emergentes y las pedagogías emergentes. El empleo de las nuevas tecnologías a nuevos objetivos educativos requiere el desarrollo de nuevas teorías, métodos, enfoques de enseñanza, evaluación y organización. Si empleamos tecnologías emergentes en educación, también hay que estar preparado para experimentar con diferentes lentes que permitan ver el mundo de diferente forma para explorar esas ideas y aplicar el conocimiento, la erudición y la colaboración. Las relaciones entre las pedagogías emergentes y las tecnologías emergentes producen un doble vínculo. Por un lado, algunas tecnologías desarrolladas sin tener una finalidad educativa específica acaban utilizándose con fines educativos y, a su vez, las prácticas pedagógicas se modifican y transforman las prestaciones tecnológicas. Adell y Castañeda (2012, 15) definen el 
concepto de pedagogías emergentes "como el conjunto de enfoques e ideas pedagógicas, todavía no bien sistematizadas, que surgen alrededor del uso de las TIC en educación y que intentan aprovechar todo su potencial comunicativo, informacional, colaborativo, interactivo, creativo e innovador en el marco de una nueva cultura del aprendizaje". Según estos autores, todavía no es posible caracterizar un corpus sistemático y los principios que subyacen en la base de las prácticas pedagógicas emergentes. Sin embargo, Adell y Castañeda (2012: 26-27) destacan algunos de los rasgos más relevantes de estas pedagogías que, en nuestra opinión, son especialmente relevantes. De este modo las pedagogías emergentes:

- Poseen una visión de la educación que va más allá de la adquisición de conocimientos o de habilidades concretas. Educar es también ofrecer oportunidades para que tengan lugar cambios significativos en la manera de entender y actuar en el mundo.

- Se basan en teorías pedagógicas ya clásicas, como las teorías constructivistas sociales y construccionistas del aprendizaje, el aprendizaje basado en proyectos, el aprendizaje trialógico, etc. y en ideas más "modernas", como el conectivismo y el aprendizaje rizomático.

- Superan los límites físicos y organizativos del aula uniendo contextos formales e informales de aprendizaje, aprovechando recursos y herramientas globales $\mathrm{y}$ difundiendo los resultados de los estudiantes también globalmente. Se anima a que los participantes conjuguen espacios y ecologías de aprendizaje.

- Muchos proyectos son colaborativos, interniveles y abiertos a la participación de docentes y alumnos de otros centros de cualquier parte del mundo e incluso de otras personas significativas.

- Potencian conocimientos, actitudes y habilidades relacionadas con la competencia "aprender a aprender", la metacognición y el aprendizaje de los estudiantes, más allá del curso, el aula, la evaluación y el currículum prescrito.

- Convierten las actividades escolares en experiencias personalmente significativas y auténticas. Estimulan el compromiso emocional de los participantes.

- Los docentes y los aprendices asumen riesgos intelectuales y transitan por caminos no trillados. Son actividades creativas, divergentes y abiertas, no mera repetición.

- En la evaluación se suele adoptar un margen de tolerancia que permite evidenciar los aprendizajes emergentes, aquellos no prescritos por el docente.

Dado que todos los componentes de las pedagogías emergentes (la tecnología, la pedagogía, el contenido, los sistemas de evaluación, etc.) están en constante evolución, los profesionales de la educación precisan estrategias de constante adaptación y cambio para entender cómo estos componentes interaccionan y modifican sus propias prácticas. De este modo, además de las características mencionadas exponemos a continuación elementos que consideramos que deben incorporar las pedagogías emergentes: 1. Las pedagogías emergentes han de apoyar el aprendizaje permanente.

La mayoría de los sistemas educativos se basan en la organización estratificada y segmentada en la que hay poca conexión entre sectores, que podría considerarse como una contribución al concepto de aprendizaje permanente. Las pedagogías emergentes han de proporcionar prácticas para apoyar el aprendizaje a lo largo de la vida. La "alfabetización para el aprendizaje" (learning literacy), es decir, la preparación de los estudiantes para autorregular y automoderar el propio proceso de aprendizaje apunta hacia el tipo de competencias transversales imprescindibles en un proceso formativo universitario, como base para el desarrollo de competencias más específicas y vinculadas al ámbito disciplinar específico que corresponda.

Entender el aprendizaje como conversación y co-creación nos lleva a considerar de forma distinta la gestión y distribución de recursos educativos: de objetos de contenido estáticos sobre una disciplina concreta, que pueden ser recuperados y estudiados, pasan a ser fragmentos multimedia de una conversación que se desarrolla de forma continua entre estudiantes y docentes a lo largo de los proyectos y tareas propuestos. 
Generar una cultura de aprendizaje activa, reflexiva y autónoma para el desarrollo de competencias entre los estudiantes implica que estos tomen más responsabilidad y decidan desde un inicio sobre su proceso formativo. Esto pasa por la negociación de los objetivos de aprendizaje, por la flexibilización de los procedimientos, los recursos y los contenidos a emplear en cada caso, así como por la apertura en el intercambio de información y el desarrollo de discusiones en torno a las tareas propuestas.

2. Las pedagogías emergentes se fundamentan en ecologías de aprendizaje La nueva ecología del aprendizaje parte de la hipótesis de que el aprendizaje es multidireccional y multi-modal $\mathrm{y}$ se entiende como parte de la vida en diferentes contextos socio-culturales y no como algo que tiene lugar exclusivamente dentro de los límites de la educación formal. Es importante trabajar en pro de la creación de relaciones abiertas, flexibles y en red a través de diversas instituciones educativas, tanto formales como informales. Tales acuerdos de trabajo intentarían reducir las barreras a la participación de todas las instituciones, aumentar las posibilidades de los estudiantes que disfrutan de experiencias educativas de alta calidad basadas en el entendimiento compartido de las historias de los alumnos y la comprensión previa.

3. Las pedagogías emergentes utilizan diferentes formas de conocimiento

Las pedagogías emergentes se basan en la metáfora de la creación de conocimiento que pone de relieve las competencias en la producción de conocimiento. En general, los sistemas (tecnológicos o no) de enseñanza y aprendizaje utilizados en las instituciones están esencialmente basados en el almacenamiento y la entrega masiva de contenidos comunes a un grupo de estudiantes. Sin embargo, este modelo no asegura el papel activo del estudiante reivindicado por los enfoques pedagógicos actuales. Según estos, el énfasis debería situarse en favorecer un flujo continuo de creación de contenidos y de comunicación en torno a los mismos, más que en su consumo y su completitud. Las tareas de aprendizaje y las herramientas utilizadas para su realización, más que los contenidos en si mismos, pueden ayudar a estudiantes a profundizar en los aprendizajes que realizan. La producción conjunta o individual de contenido, su difusión e intercambio supone una conversación dinámica de la que deriva un conocimiento generado por los propios estudiantes. El proceso de aprendizaje tiene que ver con ser capaz de conversar con uno mismo y con los demás sobre lo que se conoce. Por lo tanto, se trata más bien de poner al alcance de los estudiantes mecanismos para interactuar con múltiples servicios $\mathrm{y}$ aplicaciones que les permitan manipular, discutir y "recrear" los contenidos (Downes, 2010). Las conversaciones distribuidas que tienen lugar en torno a los contenidos de wikis, foros, blogs, etc. son ejemplos de este tipo de procesos. Los productos de estas conversaciones no solo permiten medir los aprendizajes realizados, según Downes, sino que además y como resultado de un complejo proceso interactivo, facilitan el reconocimiento del aprendizaje desde una perspectiva más competencial (lo que el estudiante es capaz de hacer).

4. Las pedagogías emergentes integran el uso de la tecnología como herramientas cognitivas

La ubicuidad de la tecnología requiere ir más allá del uso "superficial" de la tecnología, basado en la búsqueda de información. Las pedagogías emergentes alientan el uso de un nivel profundo de la tecnología, utilizando como "mindtool" o herramienta cognitiva para la creatividad, la colaboración y la productividad multimedia. La tecnología debe permitir y acelerar las relaciones de aprendizaje entre profesores y estudiantes, entre estudiantes y otros "socios de aprendizaje", como sus compañeros, mentores y personas con intereses similares.

5. Las pedagogías emergentes integran la autorregulación

Las competencias a desarrollar por los estudiantes para el aprendizaje autónomo tienen que ver con la independencia, la toma de decisiones informada, la autodirección y el control del proceso de aprendizaje, la confianza en el uso de los recursos y el entorno de aprendizaje y el desarrollo de un cierto dominio y expertez en el 
campo de estudio específico. En lugar de sistematizar el estudio a partir de módulos de contenido atomizados, se trata de tomar parte de un proceso de co-desarrollo de un contexto de aprendizaje situado, relevante y autogestionado por los propios estudiantes. Los estudiantes tienen la oportunidad de descubrir cómo se relacionan e integran entre si las unidades de contenido para acceder a perspectivas más globales. Este proceso implica una serie de decisiones sobre el tipo de materiales que analizar y producir, el tipo de herramientas a utilizar, de acciones a emprender, de redes en las que tomar parte, etc., en las que el apoyo del docente como mentor o facilitador es crucial.

6. Las pedagogías emergentes promueven actividades de aprendizaje complejas

El aprendizaje se produce enfrentando a los estudiantes a actividades de aprendizaje complejas que requieren crear y poner en práctica de manera proactiva sus conocimientos. Las tareas de las actividades complejas se guían por objetivos de aprendizaje claros y desafiantes que idealmente incorporan tanto los contenidos curriculares como los intereses o aspiraciones de los estudiantes; incluyen criterios específicos y precisos de éxito que ayudan al profesor y al alumno a saber si se están logrando los objetivos; incorporan los comentarios y los ciclos de evaluación formativa en los procesos de aprendizaje y permiten construir "confianza en sí mismo" a partir de la pro-actividad.

7. Las pedagogías emergentes son transparentes y basadas en el diseño del aprendizaje.

La pedagogía requiere hacer visible las prácticas. El razonamiento pedagógico debe ser lo más transparente posible para que pueda ser compartido entre estudiantes, profesores y otras personas involucradas en el aprendizaje de los estudiantes.

\section{Conclusiones}

Hemos sostenido que la sociedad digital está cambiando de forma substancial el acceso al conocimiento y la forma en que este se produce.
El razonamiento pedagógico debe fundamentarse en principios de diseño que permitan una descripción pedagógica completa de una intervención. Debe incluir los criterios de diseño, las propiedades de las actividades de enseñanza-aprendizaje, y las capacidades de las herramientas y la tecnología convencional y digital que se utiliza.

De acuerdo con la definición de Conole (2013: 186) el diseño del aprendizaje puede entenderse como una metodología pedagógicamente fundamentada y basada en el uso apropiado de recursos y tecnologías, que permite tomar decisiones más informadas en el diseño de intervenciones educativas. Esto abarca desde el diseño de recursos o de actividades de aprendizaje individuales hasta el diseño curricular. Uno de los principios en que se basa esta metodología es en hacer el proceso de diseño más explícito y fácil de compartir. El diseño del aprendizaje es también un campo de investigación y desarrollo, en la medida que se basa en el análisis de evidencias empíricas, que ayudan a comprender el proceso de diseño del aprendizaje y a la vez se propone el desarrollo de recursos, herramientas y estrategias que faciliten la propia tarea de diseño.

El campo del diseño del aprendizaje se ha desarrollado en los últimos años y ahora ofrece un conjunto de métodos, herramientas, sistemas y modelos (Craft y Mor, 2012) que puede empoderar a los docentes en el diseño de escenarios que proporcionen experiencias de aprendizaje más ricas. Se trata todavía de un campo de conocimiento poco desarrollado, sistematizado y estandarizado, y en consecuencia, cuenta a día de hoy con un número relativamente bajo de herramientas e instrumentos de representación, y por lo tanto con un lenguaje todavía limitado como apoyo en el desarrollo y compartición de las tareas de diseño.

La sociedad disciplinaria se va transformando en una sociedad en que los espacios no están delimitados y las actividades, la formación 
y el aprendizaje dependen cada vez más del propio sujeto.

La consecuencia de la caída de los muros del conocimiento tiene implicaciones importantes para los profesionales de la educación. Las personas precisan una formación que les ayude a vivir en esta sociedad sin muros evitando la fragmentación, dispersión y un exceso de carga emocional y cognitiva. Para ello, es muy importante dotar a la persona de capacidades para la autorregulación del aprendizaje.

Lossistemasdeformacióntradicionalesbasados en metodologías didácticas muy centradas en lo disciplinar precisan transformarse $\mathrm{y}$ combinarse con planteamientos que orienten en el diseño de estos nuevos espacios a través

\section{Referencias}

Adell, J. y Castañeda, L. (2012). Tecnologías emergentes, ¿pedagogías emergentes? En J. Hernández, M. Pennesi, D. Sobrino y A. Vázquez (coord.). Tendencias emergentes en educación con TIC. Barcelona: Asociación Espiral, Educación y Tecnología. págs. 13-32.

Attwell, G. (2007). The Personal Learning Environments - the future of eLearning? eLearning Papers, 2(1), 1-8.

Bartolomé, A. \& Steffens, K. (2011). Technologies for self-regulated learning. En R. Carneiro, P., Lefrere, K., Steffens, K. \& Underwood, J. (Eds.), Self-regulated Learning in Technology Enhanced Learning Environments: A European Review (pp. 2131). Rotterdam: Sense Publishers.

Bereiter, C. (2002). Education and mind in knowledge age. Hillsdale: N.J, Lawrence Erlbaum associates.

Burbules, N. C. (2014). El aprendizaje ubicuo: nuevos contextos, nuevos procesos. Entramados: educación y sociedad, 1(1), 131134.

Conole, G. (2013). Las pedagogías de los entornos personales de aprendizaje. En de experiencias basadas en la interacción social, la participación activa y los entornos complejos. La pedagogía como ciencia del diseño es un camino que conviene explorar y desarrollar. Fundamentar lo pedagógico en el diseño implica un razonamiento pedagógico más transparente que permite el diálogo, la participación y la modificación dinámica de los escenarios de aprendizaje. El papel de las tecnologías digitales es fundamental pero deja de tener sentido preguntarse sobre si estas proporcionan un mayor aprendizaje, ya que resulta imposible prescindir de lo tecnológico. Se trata, eso sí, de dar coherencia a los diseños visibilizando el diseño pedagógico frente a los recursos digitales.

L. Castañeda y J. Adell (Eds.), Entornos Personales de Aprendizaje: Claves para el ecosistema educativo en red (pp. 185-188). Alcoy: Marfil.

Cope, B., \& Kalantzis, M. (2010). "Multialfabetización": nuevas alfabetizaciones, nuevas formas de aprendizaje. Boletín de la Asociación Andaluza de Bibliotecarios, 25(98), 53-92.

Dewey, J. (1975). Democracia y Educación. Madrid: Morata.

Downes, S. (2010). New technology supporting informal learning. Journal of Emerging Technologies in Web Intelligence, 2(1), 27-33. http://dx.doi.org/10.4304/ jetwi.2.1.27-33

Freire, J. y Schuch Brunet, K. (2010) Políticas y prácticas para la construcción de una universidad digital. La Cuestión Universitaria, 6. Boletín Electrónico de la Cátedra UNESCO de Gestión y Política Universitaria, UPM, 2: 85-94.

Han, B-C. (2012). La sociedad del cansancio. Barcelona: Herder. 
Han, B-C. (2014). Psicopolítica. Barcelona: Herder.

Innerarity, D (2011). La democracia del conocimiento. Por una Sociedad inteligente. Barcelona: Paidós.

Jokisalo, E. y Riu, A. (2009). Informal learning in the era of Web 2.0. eLearning Papers. $14(5)$.

Luhmann, N (1998). Sistemas sociales.
Lineamientos para una teoría general. Barcelona: Anthropos.

Mor, Y., \& Craft, B. (2012). Learning design: reflections upon the current landscape. Research in learning technology, 20. http://dx.doi.org/10.3402/rlt.v20i0.19196

Sharples, M, et al. (2012), Innovating Pedagogy makers. Milton Keyne: Open University.

Siemens, G. (2006). Connectivism: Learning theory or pastime of the self-amused. Manitoba, Canada: Learning Technologies Centre.

Siemens, G. (2010). Conociendo el conocimiento. Ediciones Nodos Ele.

Suárez, C., (2012). De la escuela-lugar a la escuela-nodo. Blog sobre Educación y Virtualidad. Recuperado el 20 diciembre de 2014.

http://educacion-virtualidad.blogspot.com. es/2012/03/de-la-escuela-lugar-la-escuelanodo.html

Veletsianos, G. (2010). A definition of emerging technologies for education. En Veletsianos, G. (ed.) Emerging technologies in distance education (pp. 3-22). Athabasca, CA: Athabasca University Press.

Wild, F., Mödritscher, F., \& Sigurdarson, S. (2008). Designing for change: mash-up personal learning environments. eLearning Papers, 9. 